This item was submitted to Loughborough's Research Repository by the author.

Items in Figshare are protected by copyright, with all rights reserved, unless otherwise indicated.

\title{
Molecular dynamics simulations of nanoindentation and nanotribology
}

PLEASE CITE THE PUBLISHED VERSION

http://dx.doi.org/10.1098/rsta.2005.1621

\section{PUBLISHER}

(c) The Royal Society

\section{VERSION}

AM (Accepted Manuscript)

\section{LICENCE}

CC BY-NC-ND 4.0

\section{REPOSITORY RECORD}

Kenny, Steven D., Devianee Mulliah, Carlos F. Sanz-Navarro, and Roger Smith. 2019. "Molecular Dynamics Simulations of Nanoindentation and Nanotribology". figshare. https://hdl.handle.net/2134/11637. 
This item was submitted to Loughborough's Institutional Repository (https://dspace.lboro.ac.uk/) by the author and is made available under the following Creative Commons Licence conditions.

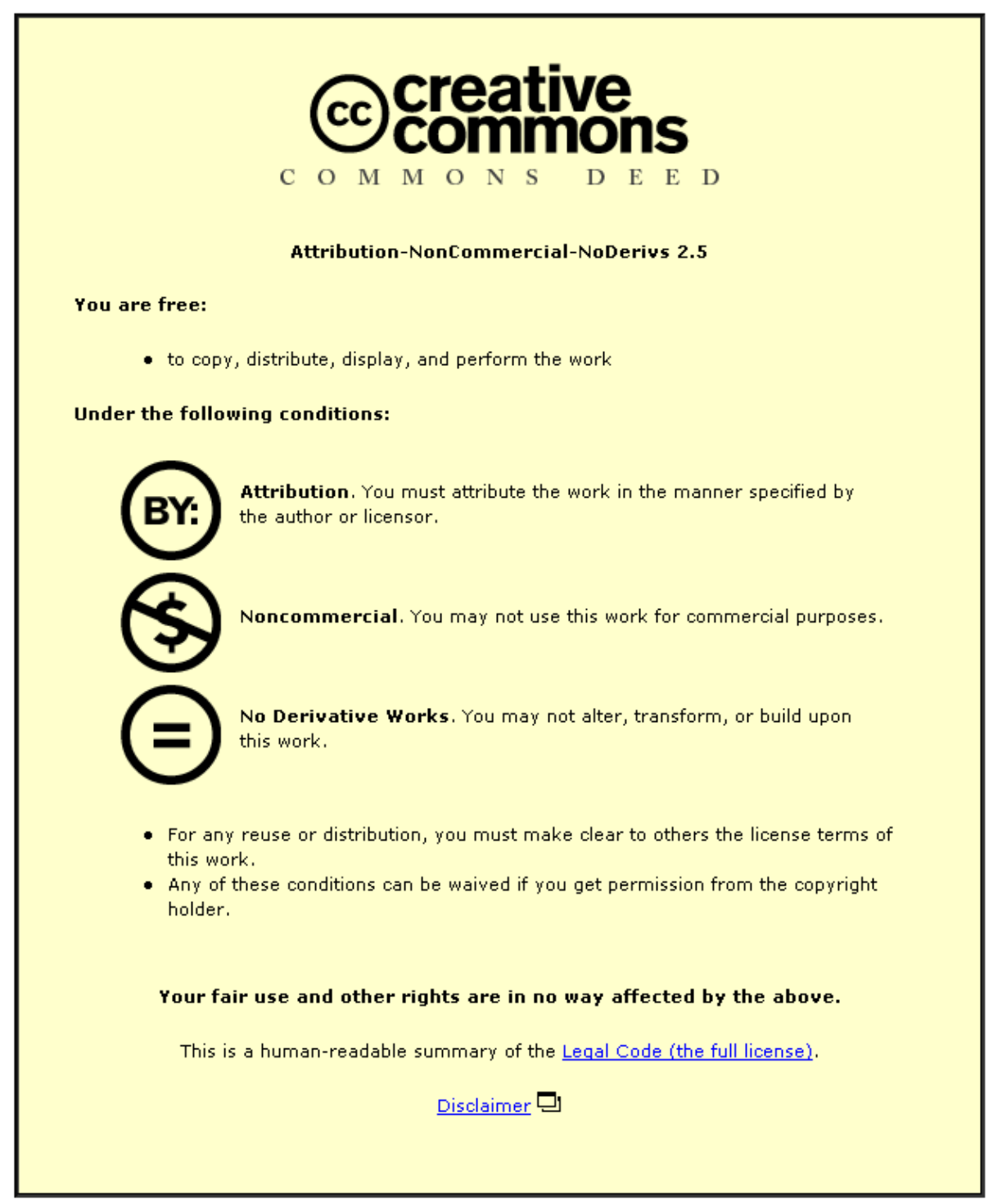

For the full text of this licence, please go to: http://creativecommons.org/licenses/by-nc-nd/2.5/ 


\title{
Molecular Dynamics simulations of Nanoindentation and Nanotribology
}

\author{
By S. D. Kenny, D. Mulliah, C. F. Sanz-Navarro and Roger Smith \\ Modelling of Materials Group, Department of Mathematical Sciences, \\ Loughborough University, Loughborough, Leicestershire LE11 3TU, UK
}

We present results of parallel molecular dynamics (MD) simulations of nanoindentation and nanotribology experiments. The models we have developed describe both the sample and the indenter atomistically and model the effect of the cantilevers in an atomic force microscope (AFM) through the use of springs. We show that the simulations are in good qualitative agreement with experiment and help to elucidate many of the mechanisms that take place during these processes. In particular we illustrate the role that dislocations play both in nanoindentation and also in stick-slip. Further to this we show how real-time visualisation and computational steering have been employed in these simulations to capture the dynamical events that take place.

Keywords: Molecular dynamics, nanoindentation, nanotribology

\section{Introduction}

Nanoindentation and nanotribology methodologies are recent techniques for the study of materials on the nanoscale. They utilise new experimental equipment such as the atomic force microscope (AFM) and quartz crystal microbalance in order to gain a measure of control that was previously unachievable. These techniques give us the opportunity to understand materials that have features on the nanoscale, where more traditional methods are inappropriate. There are a number of new materials with such features, including nanoparticulate materials and thin film coatings for which these nano-techniques are especially appropriate. These methodologies are not as well understood as traditional mechanical testing techniques due to the direct influence that atomistic scale processes have on them. However, they are potentially more powerful since they allow the dynamic response of the material to be investigated. Atomistic simulations have been applied to model these methodologies to help to elucidate the dynamical events that take place (Christopher et al. 2001; Smith et al. 2003, Mulliah et al. 2004a,b).

These nano-techniques are able to probe mechanisms that occur in a material at the atomistic level. Thus the effect of events such as dislocation emission can be directly observed in the experimental results and elucidated through the use of modelling (Smith et al. 2003). Additionally they can be used to study phase transformations that occur under pressure (Sanz-Navarro et al. 2004a). The methodologies can also be used to study the stick-slip phenomena at an atomistic scale (Mulliah et al. 2004b). 


\section{Methodology}

The nanoindentation and nanotribology studies both employ atomistic substrates and indenters. The indenters used were constructed from a diamond cube by cutting along $\{111\}$ planes (Christopher et al. 2001). This gives a tip whose sides are $\{100\}$ planes and with the plane perpendicular to the indentation direction being the (111) plane. The tips were then blunted by rounding the tip in order to more accurately model the experiments. The tips were then fully relaxed before being used in the simulations as the faces and edges of the tip reconstruct. A number of different tips were used in the simulations in order that the tip size is consistent with the substrate being employed.

For the indentation simulations, the tip was attached to a spring which was connected to a support. This support was moved down towards the sample with a fixed speed, as shown in figure 1 (a). The support was then pulled away from the surface at the end of the indentation phase to simulate tip retraction. The use of the springs and supports models the effect of the cantilever in the AFM employed for these studies. The nanotribology studies use a similar model which incorporates additional springs and supports in the horizontal directions, figure 1 (b). In this case indentation happens as before but after insertion the indenter was first kept fixed to allow relaxation to occur before being pulled across the surface through the use of one of the horizontal springs. The additional horizontal spring and the vertical spring are employed during the scratching phase of the simulation which allows the indenter to move in these directions during this phase. Spring constants were chosen to be such that they are consistent with experimental values and so that they provide a reasonable behaviour for the tip. The equations of motion for the fixed atoms in the indenter during indentation are given by

$$
m a_{y}(t)=F_{y}(t)+k_{y}\left(y_{\mathrm{B}}(t)-y(t)\right)
$$

and during scratching by

$$
\begin{aligned}
& m a_{x}(t)=F_{x}(t)+k_{x}\left(V_{\mathrm{A}} t-x(t)\right) \\
& m a_{y}(t)=F_{y}(t)+k_{y}\left(y_{\max }-y(t)\right) \\
& m a_{z}(t)=F_{z}(t)-k_{z} z(t) .
\end{aligned}
$$

With $y_{B}(t)$ referring to the position of the support $\mathrm{B}, V_{A}$ being the velocity of support A during the scratching phase and $y_{\max }$ being the maximum distance moved by support B during the indentation phase.

Integration was performed using a velocity-Verlet algorithm (Allen \& Tildesley 2000) with a time step of 1 fs used throughout the simulations. For the nanoindentation simulations, the support was moved at a speed of $53.33 \mathrm{~m} / \mathrm{s}$ towards the surface for a period of $75 \mathrm{ps}$ before being retracted. The nanoindentation simulations used Lindhard-Scharff damping (Lindhard et al. 1963) to remove excess energy from the system, so as to prevent local heating occurring. For the nanotribology simulations the tip was indented into the surface at a speed of $10 \mathrm{~m} / \mathrm{s}$. The tip support was then moved at a speed of $1 \mathrm{~m} / \mathrm{s}$ for the scratching part of the simulation. The nanotribology modelling was carried out at a temperature of $300 \mathrm{~K}$ with the substrate and the tip initially heated to this temperature and then maintained at this temperature by employing a Nosé-Hoover (Nosé 1984; Hoover 1985) thermostat on the 


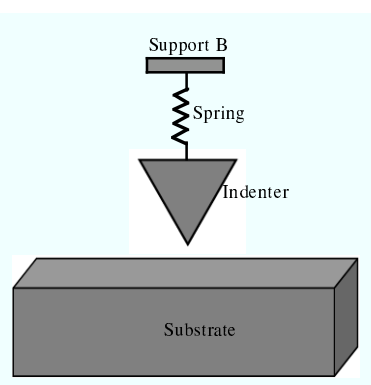

(a)

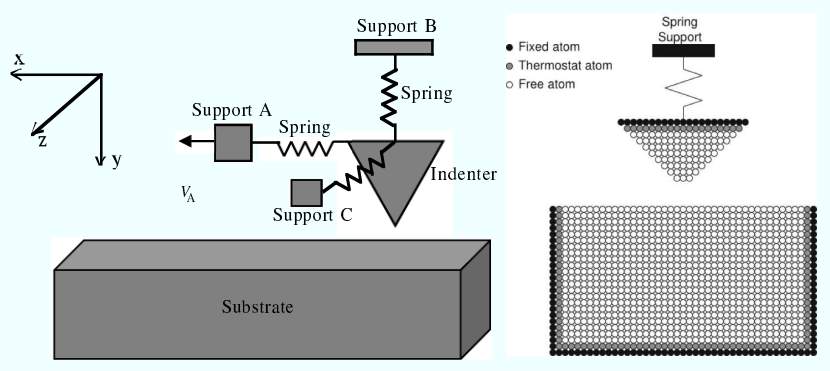

(b)

(c)

Figure 1. The setup for the tip and the substrate for the (a) nanoindentation model, (b) nanotribology model. Springs are attached to the supports which are moved to simulate an AFM. The vertical direction is referred to as the $y$ direction with the direction of scratching being the $x$ direction. (c) shows the boundary conditions for the simulations performed at $300 \mathrm{~K}$.

boundary atoms, as shown in figure 1 (c). Excessive local heating in this case was controlled through the use of a slow scratching speed. Tests have shown that the local heating that occurs with these conditions is very small (less than 10 degrees). This is an improvement on earlier models which were carried out at 0K (Mulliah et al. 2004b) and some differences are observed.

The interactions between the $\mathrm{C}$ atoms in the indenter were modelled using the Brenner potential (Brenner 1990, 1992). For the substrate the Ag-Ag interactions were described by the Ackland potential (Ackland et al. 1987). The interactions between the indenter atoms and the substrate atoms were modelled using a ZBL potential (Biersack et al. 1985), which is purely repulsive.

The simulations have been analysed through the use of a number of different filtering techniques during visualisation (Sanz-Navarro et al. 2004). In this work we have mainly used the modulus of the slip vector filtering (Rodríguez de la Fuente et al. 2002) which illustrates atoms that either lie on a stacking fault or a full dislocation, thus allowing the understanding of the nature of plastic deformation in the material. The slip vector is defined by

$$
\mathbf{s}=\frac{1}{n_{s}} \sum_{\beta=1}^{n_{s}}\left(\mathbf{r}_{t}^{\beta}-\mathbf{r}_{0}^{\beta}\right)
$$

where $n_{s}$ is the number of slipped neighbours of such an atom, $\beta$ indexes those neighbours and $\mathbf{r}_{t}^{\beta}$ and $\mathbf{r}_{0}^{\beta}$ denote the relative vector between the given atom and its neighbour $\beta$ at the current and initial times respectively. We have also used height filtering to illustrate regions where the height of the surface has been raised due to dislocation emission. All of this work has been performed using Loughborough real-time visualisation tool, LBOVIS, which allows us to study the evolution of the system as the simulation is running.

The RealityGrid computational steering library (Pickles et al. 2004a,b) was employed in the simulations. As well as facilitating the steering of the code the library also enabled the real-time visualisation of the simulations. Steering data was communicated through the use of a simple object access protocol (SOAP) 


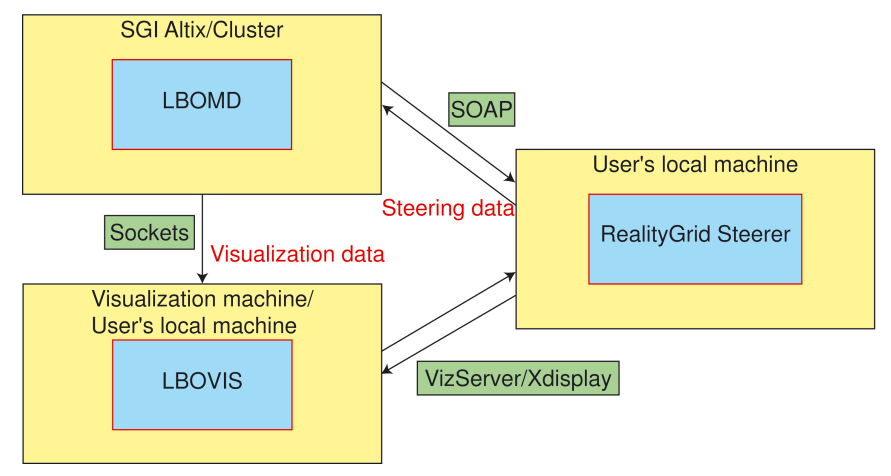

Figure 2. The connections between the components of the codes used when performing real-time visualisation and steering of an MD simulation.

repository whilst the visualisation data was communicated using sockets, with the initial communication for the connection of the sockets occurring through the use of SOAP. Figure 2 illustrates the connections between the various components in the system; LBOMD is the MD code employed, LBOVIS is the visualisation code and the RealityGrid steerer is the steering client used. Steering in these simulations was mainly used to rewind a simulation to study dynamical events in greater detail and to monitor the simulation parameters in order to check quantities such as the energy conservation.

A number of experimentally measurable quantities have been extracted from the simulations so as to compare the results obtained with the literature. In particular the nanohardness of the surface has been calculated using

$$
H=\frac{F_{\max }}{\sigma}
$$

where $F_{\max }$ is the exerted force at the peak load, $\sigma$ is the cross-sectional area of the indent approximated by $(3 \sqrt{3} / 2) h_{\max }^{2}$ and $h_{\max }$ is the indenter penetration at the peak load.

\section{Nanoindentation Simulations}

Six MD simulations of the nanoindentation of Ag surfaces have been performed in order to study three different surfaces, (100), (110) and (111), using two different indenter orientations. The system size for all three substrates was $300 \AA \times 300$ $\AA \times 300 \AA$, containing around eight hundred thousand atoms. The cube corner indenter was used such that in one of the orientations, one of its sides was parallel to the substrate edge and in the other, it was at $45^{\circ}$ to the substrate edge. A $3.5 \AA$ layer of atoms on the sides and the base of the substrate were frozen in position to prevent the substrate moving away from the indenter during indentation. Emitted dislocations move out as far as the boundaries during the simulations, however, this has little effect on the simulations. This can be seen by observing at the stress at the boundary during the simulations and by monitoring the calculated force-depth curves. The vertical spring used in the simulation had a spring constant of 1282 


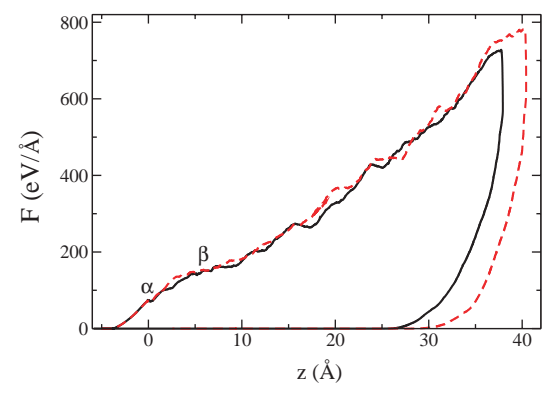

Figure 3. The force depth curves for the indentations of the (100) Ag surface. The point where the initial plastic deformation takes place is marked by $\alpha$, with the point where the first dislocation that moves parallel to the plane of the surface is emitted being marked by $\beta$.

$\mathrm{Nm}^{-1}$, which was found to give a good balance between spring compression and indentation whilst being consistent with typical values for AFM cantilevers.

The results from the indentation of all three surfaces show evidence of large amounts of dislocation formation. In fact in all cases, dislocations emission is visible shortly after the tip touches the surface. Dislocations observed are a mixture of Shockley partial dislocations, with Burgers vector $\frac{1}{6}<112>$ and full dislocations, with Burgers vector $\frac{1}{2}<110>$.

Figure 3 shows the force-depth curves for the indentations using the two different indenter orientations into the (100) Ag surface. In both cases an initial plastic deformation can be seen shortly after the tip penetrates into the surface, marked with $\alpha$. Visualisation of the surface at this point reveals that a small pyramid of atoms lying directly under the indenter have slipped as can be seen in figure 4 (a). Inspecting this system using the slip vector defined in equation 2.5 shows the formation of a Shockley partial dislocation, figure 4 (b). After a further 1 ps of simulation the other two sides of the sunk pyramid detach giving rise to a second Shockley partial that travels in the opposite direction through the lattice.

When the indenter has reached a depth of $6 \AA$ into the (100) surface, a v-shaped dislocation is emitted which moves parallel to the surface plane. The appearance of a raised area on the surface coincides with the emission of this dislocation. Further to this a low hillock is seen to propagate across the surface as this dislocation is emitted. This is due to the fact that this dislocation injects an additional half plane of atoms into the material causing the surface atoms to be pushed up. These hillocks are also observed experimentally (Rodríguez de la Fuente et al. 2002) and can become quite pronounced with deeper indentation depths. The effect that the emission of multiple dislocations of this type has on the height of the surface can be seen in figure 5 . Figure 5 (a) shows the dislocations that have been emitted and figure 5 (b) shows the amount that the surface is raised due to these dislocations. For the (100) surface the majority of slip occurs in the $4\{111\}$ planes that are at $54.7^{\circ}$ to the surface. Slip in these planes moves in the $4<110>$ directions that are parallel to the surface plane.

Figure 6 shows the force depth curves for the three different surfaces studied 


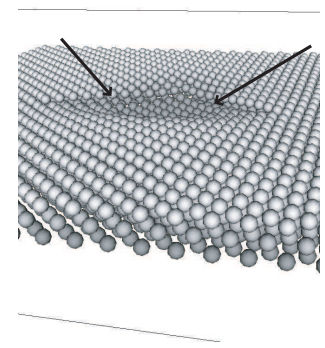

(a)

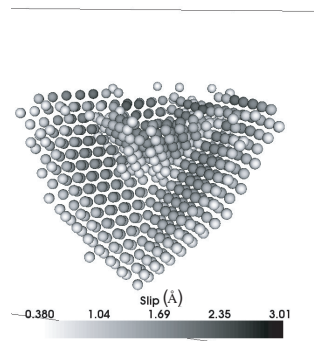

(b)

Figure 4. The onset of plastic deformation during the indentations of the (100) Ag surface. (a) shows the atoms near the surface and it can be seen that on two sides of the square on the surface atoms have displaced downwards by a lattice parameter; (b) shows the slip vector for the atoms and from it the existence of Shockley partial dislocation can be seen. This is shown by the dark atoms on the same sides as is indicated by the arrows.

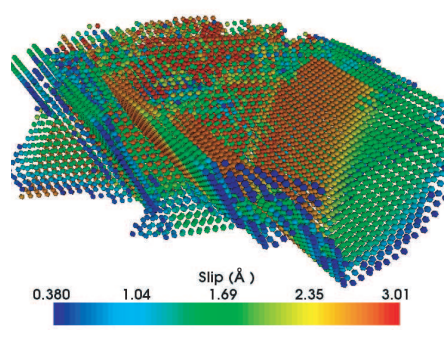

(a)

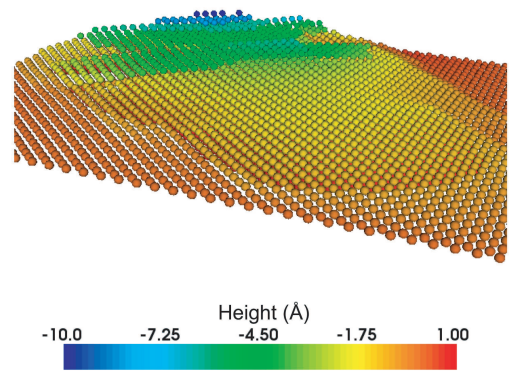

(b)

Figure 5. Images illustrating the hillocks formed on the Ag (100) surface during indentation (a) uses the slip vector filtering given in 2.5 to visualise the dislocations; (b) shows the height of the atoms above the original surface. The slipped atoms are sitting on a $\{111\}$ plane and moving in a $<110>$ direction. Movies available in online version.

and for both indenter orientations. It can be seen that in general the surface and indenter orientation have little influence on the force depth curves. This is not true, however, for the indentation of the (111) surface with indenter orientation 1 . In this case the material appears to harden after an indentation to a depth of approximately $30 \AA$. This is also reflected in the calculated values for the nanohardness, given in table 1 . If we compare the number of atoms with slip vectors lying between $1.5 \AA$ and $3 \AA$, these are the atoms that are part of dislocation loops and for the (111) surface there are almost twice as many in the case of orientation 1 as for orientation 2. In contrast, this value for the (100) and (110) surfaces is very similar for both indenter orientations. This implies that the hardening that is observed is due to the interaction of dislocations. 

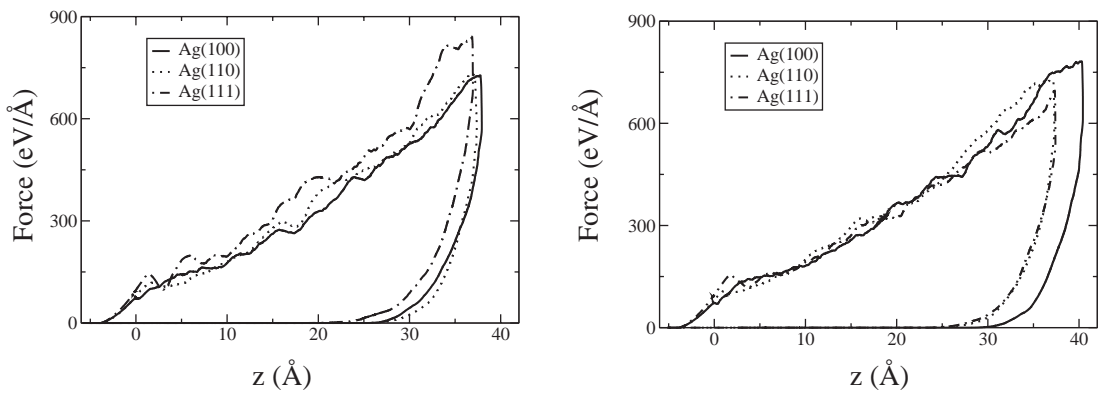

Figure 6. Graphs showing the force depth curves for the three different surfaces studied, (100), (110) and (111), with the two different indenter orientations.

Table 1. Hardness values for the three different surfaces.

(Hardness values as calculated using equation 2.6 for the three different surfaces studied and the two different indenter orientations.)

\begin{tabular}{|c|c|c|}
\hline $\begin{array}{c}\text { surface } \\
\text { orientation }\end{array}$ & $\begin{array}{c}\text { orientation I } \\
(\mathrm{GPa})\end{array}$ & $\begin{array}{c}\text { orientation II } \\
(\mathrm{GPa})\end{array}$ \\
\hline$(100)$ & 32 & 30 \\
$(110)$ & 32 & 32 \\
$(111)$ & 38 & 31 \\
\hline
\end{tabular}

\section{Nanotribology Simulations}

We have performed two simulations of nanotribology where we indent into the (100) surface of $\mathrm{Ag}$, one to a depth of $5 \AA$ the other to a depth of $15 \AA$. For both cases a substrate of size $180 \times 42 \times 122 \AA$ containing 54,000 atoms was used. For the simulations performed at an indentation depth of $5 \AA$, the spring constants of the horizontal, vertical and lateral springs used in the calculations were $k_{x}=40.05$ $\mathrm{Nm}^{-1}, k_{y}=240.30 \mathrm{Nm}^{-1}$ and $k_{z}=240.30 \mathrm{Nm}^{-1}$ respectively, whereas for the simulation at the deeper indent of $15 \AA$ the spring constants were $k_{x}=120.15$ $\mathrm{Nm}^{-1}, k_{y}=352.44 \mathrm{Nm}^{-1}$ and $k_{z}=352.44 \mathrm{Nm}^{-1}$. The mass of the tip was taken to be $3.14 \times 10^{-23} \mathrm{~kg}$. The values of the vertical spring constants were chosen to be consistent with values in an AFM and also so that the springs did not elongate or compress excessively during a simulation.

The nanotribology simulations for the Ag system include the effects of finite temperature into the simulation in contrast to our earlier work (Mulliah 2004a, b). Calculated values of the friction coefficients, given in table 2, are similar to those from the earlier work when the actual indentation depths are comparable. The actual indentation depths, i.e. those of the tip apex rather than the support, reached during the indentation phase of these simulations were 2.35 and $10.67 \AA$ for the cases where the support has moved such as to indent to a depth of 5 and 15 $\AA$ respectively. These compare to the $0 \mathrm{~K}$ results of 1.25 and $9.86 \AA$ respectively. This means that we cannot directly compare the shallower indent results but for the case of the deeper indent the corresponding values for the dynamic and static 
Table 2. Results for the nanotribology simulations of Ag.

(Calculated friction coefficients for the case of $\mathrm{Ag}$ for the indentation depths of 5 and 15 Å.)

\begin{tabular}{|c|c|c|c|c|c|}
\hline $\begin{array}{c}\text { Indentation } \\
\begin{array}{c}\text { Depth } \\
\AA\end{array}\end{array}$ & $\begin{array}{c}\text { Normal } \\
\text { force } \\
(\mathrm{nN})\end{array}$ & $\begin{array}{c}\text { Frictional } \\
\text { force } \\
(\mathrm{nN})\end{array}$ & $\begin{array}{c}\text { Dynamic } \\
\text { friction } \\
\text { coefficient }\end{array}$ & $\begin{array}{c}\text { Static } \\
\text { friction } \\
\text { coefficient }\end{array}$ & $\begin{array}{c}\text { Height } \\
\text { of pile-up } \\
(\AA)\end{array}$ \\
\hline 5 & 36.08 & 8.21 & 0.228 & 0.324 & 3.76 \\
\hline 15 & 106.29 & 43.20 & 0.406 & 0.500 & 9.00 \\
\hline
\end{tabular}

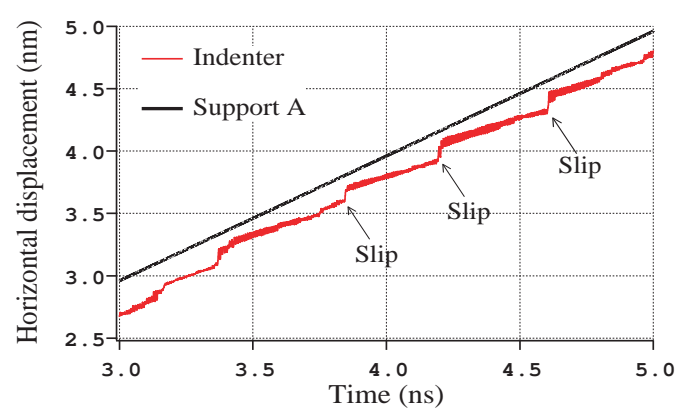

(a)

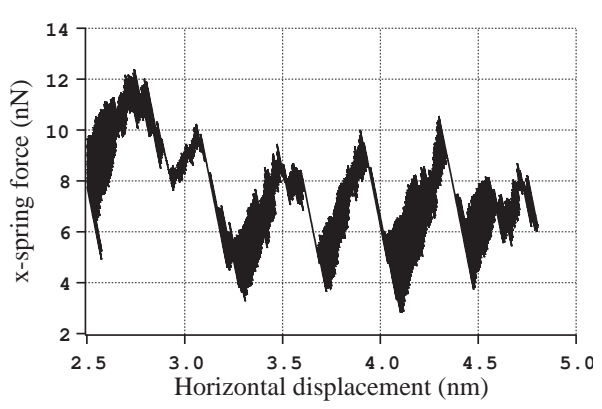

(b)

Figure 7. Graphs showing (a) the horizontal motion of the indenter and the support during the scratching phase and (b) the horizontal spring force of the indenter during the scratching, both graphs are from simulations with an indentation depth of $5 \AA$.

friction coefficients were 0.46 and 0.54 respectively. Thus it seems that the friction coefficients are reduced slightly at $300 \mathrm{~K}$ over the $0 \mathrm{~K}$ results.

During the scratching phase, stick-slip events are seen for both indentation depths, which can be seen in the graphs in figure 7 (a) and (b), where a number of the slip events have been marked with an arrow. The behaviour seen in the graphs is similar to that observed for the simulations at $0 \mathrm{~K}$. However, when we investigate the dynamical events responsible for the behaviour, marked differences are found. The first difference is the timescale over which they occur. Dislocations in the system are emitted and retracted on a timescale that is quicker than that over which snapshots would typically be taken. The dislocations are retracted after the tip has slipped since the tip no longer causes strain in the relevant slip system. Due to this the computational steering tools were found to be invaluable for rewinding simulations and studying these dynamical events in far greater detail. Figure 8 shows a series of snapshots from the simulation at an indentation depth of $5 \AA$ where the emission and retraction of a number of dislocations can be observed. This highlights a second difference to the $0 \mathrm{~K}$ work in that most dislocations that are emitted are Shockley partial dislocations and these are retracted once the indenter moves past the point from which they were emitted. This is in contrast to earlier studies where a mixture of Shockley partial dislocations and full dislocations were observed. 


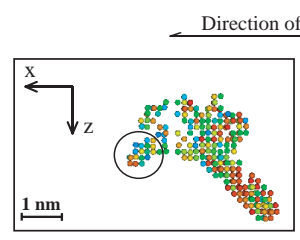

(a) $\mathrm{t}=1.4 \mathrm{~ns}$

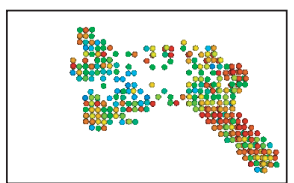

(d) $\mathrm{t}=3.543 \mathrm{~ns}$

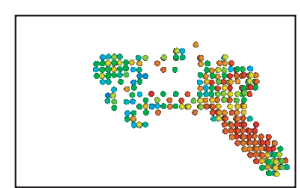

(b) $\mathrm{t}=3.46 \mathrm{~ns}$

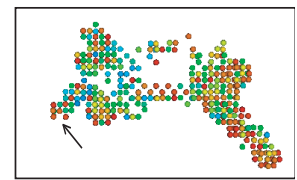

(e) $\mathrm{t}=3.8 \mathrm{~ns}$

slip vector $(\AA)$

$\begin{array}{lllll}0.38 & 1.04 & 1.70 & 2.35 & 3.0\end{array}$

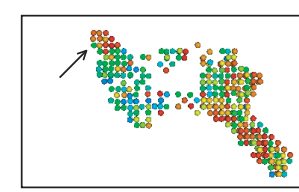

(c) $\mathrm{t}=3.496 \mathrm{~ns}$

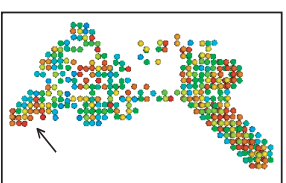

(f) $\mathrm{t}=5.0 \mathrm{~ns}$

Figure 8. Dislocation emission during the scratching of the (100) Ag surface with an indentation depth of $5 \AA$. In (a) a Shockley partial dislocation is circled, which retracts as can be seen in (b). This followed by the emission of further dislocations, marked by the arrows, in (c), (e) and (f). Movie available in online version.

\section{Conclusions}

We have shown that during both nanoindentation and nanotribology dislocations play an important role in fcc Ag. Furthermore in the case of nanoindentation we have shown that the stepped terraces observed in nanoindentation experiments on the (100) surfaces of fcc materials are due to the emission of multiple dislocations that move parallel to the surface. These dislocations each contribute to a raising of the surface height and the steps come from the fact that the outer dislocations enclose the inner ones, as shown in figures 5 (a) and (b).

We have also shown the effect that finite temperature has on the behaviour of dislocations in the case of the nanotribology modelling of Ag. This has highlighted two main differences over models run at $0 \mathrm{~K}$; namely the predominance of Shockley partial dislocations in the finite temperature simulations compared to the mix of Shockley partial dislocations and full dislocations observed at $0 \mathrm{~K}$. This means that the majority of the dislocations emitted are retracted during the scratching phase for the finite temperature case. We only ever observe the full retraction of partial dislocations. The second feature is the timescale over which the dislocation emission and retraction takes place in the finite temperature simulations. This is much faster than was observed during the $0 \mathrm{~K}$ simulations. The dislocation emission and retraction in this system occurs over such a timescale that the traditional approach of writing output every, say, 1000 timesteps is likely to miss completely these dynamical events. Here we have shown how one can use computational steering to study these events in greater detail and capture the important processes. 


\section{Acknowledgements}

We would like to acknowledge funding from EPSRC, Grant No. GR/R67699/02 and GR/R18581/01. Calculations were performed using the facilities of the Loughborough's high performance computer and visualisation centre. We would like to thank the SVE group at Manchester computing for useful discussions on the implementation of the RealityGrid computational steerer.

\section{References}

Ackland, G. J., Tichy, G., Vitek V. and Finnis, M. W. 1987 Simple N-body potentials for the Noble-metals and Nickel. Phil. Mag. A56, 735-756.

Allen, M. P. and Tildesley, D.J. 2000 Computer Simulation of Liquids. New York: Oxford University Press.

Biersack, J. P., Ziegler, J., Littmack, U. 1985 The Stopping and Range of Ions in Solids. Oxford: Pergamon press.

Brenner, D. W. 1990 Empirical potential for hydrocarbons for use in simulating the chemical vapor-deposition of diamond films. Phys. Rev. B42, 9458-9471.

Brenner, D. W. 1990 Correction. Phys. Rev. B46, 1948-1948.

Christopher, D., Smith, R. and Richter, A. 2001 Nanoindentation of carbon materials. Nucl. Inst. Meth. B180, 117-124.

Hoover, W. G. 1985 Canonical dynamics - equilibrium phase-space distributions. Phys. Rev. A31, 1695-1697.

Lindhard, J., Scharff, M. and Schiøtt, H. E. 1963. Mat. Fys. Medd. Dan. Vid. Selsk. 33 14.

Mulliah, D., Kenny, S. D., Smith, R. and Sanz-Navarro, C. F. 2004a Molecular dynamic simulations of nanoscratching of silver(100). Nanotechnology 15, 243-249.

Mulliah, D., Kenny, S. D. and Smith, R. $2004 b$ Modeling of stick-slip phenomena using molecular dynamics. Phys. Rev. B69, 205407.

Nosé, S. 1984 A molecular-dynamics method for simulations in the canonical ensemble. Mol. Phys. 52, 255-268.

Pickles, S. M., Pinning, R. L., Porter, A. R., Riley, G., Ford, R., Mayes, K., Snelling, D., Stanton, J., Kenny, S. D. and Jha, S. $2004 a$ The RealityGrid computational steering API. http://www.sve.man.ac.uk/Research/AtoZ/RealityGrid/Steering/ReG_steering_api.pdf.

Pickles, S. M., Haines, R., Pinning, R. L. and Porter, A. R. $2004 b$ Practical tools for computational steering. In Proc. of the UK e-Science All Hands Meeting, Nottingham, UK, September 2004, paper 201.

Rodríguez de la Fuente, O., Zimmerman, J. A. , Gonzlez, M. A., de la Figuera, J., Hamilton, J. C., Pai, W. W. and Rojo, J. M. 2002 Dislocation emission around nanoindentations on a (001) fcc metal surface studied by scanning tunneling microscopy and atomistic simulations. Phys. Rev. Lett. 88, 036101.

Sanz-Navarro, C. F., Kenny, S. D., and Smith, R. 2004a Atomistic simulations of structural transformations of silicon surfaces under nanoindentation. Nanotechnology 15, 692-697.

Sanz-Navarro C. F., Kenny, S. D., Porter, A.R. and Pickles, S.M. 2004 Real-time visualization and computational steering of molecular dynamics simulations of materials science. In Proc. of the UK e-Science All Hands Meeting, Nottingham, UK, September 2004 , paper 107.

Smith, R., Christopher, D., Kenny, S. D., Richter, A. and Wolf, B. 2003 Defect generation and pileup of atoms during nanoindentation of Fe single crystals. Phys. Rev. B67, 245405. 